\title{
A SIMPLE FAT EXTRACTOR.
}

By G. A. STOKES.

(Read at the Meeting, May 6, 1914.)

Most of the fat extractors at present in use appear to have the following defects: (1) The substance to be extracted is placed in a glass tube, so that the outer surface is not exposed immediately to the action of the solvent. (2) The solvent usually exerts its action after it has been cooled by condensation. (3) The extractors are more or less complicated and therefore expensive.

The apparatus I have devised consists of a flask, a piece of wire, and a thimble of fat-free paper. Through the cork connecting the flask with the condenser passes a wire, so bent as to hold a fat-free paper thimble within the flask. Into this thimble the substance to be extracted is placed, and above it a layer of fat-free cotton-wool to prevent any of the substance floating over. The wire is then bent into a spring so as to allow the thimble to be inserted and the cotton-wool pressed down. At the commencement of the extraction the thimble and its contents are forced down by the wire so that they are immersed in the solvent.

After boiling for a short time in the solvent, the hook of the wire on the outside is raised, without disconnecting any part of the apparatus, and the thimble brought out of the liquid, into the neck of the flask. As the space between the neck and thimble is narrow, the boiling vapour as it ascends must act upon the outside of the thimble. The thimble being pervious is constantly being washed inside and out by the hot solvent.

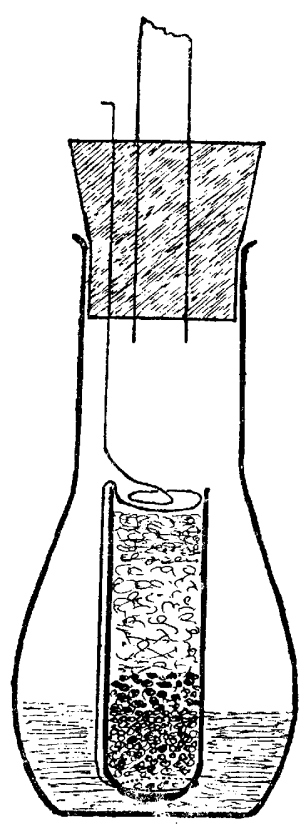

When the extraction is finished, the flask is detached, the solvent evaporated, and the flask and its contents weighed. 
296 NOTE ON "SHREWSBURY'S REACTION FOR PARAFFIN"

The advantages claimed are speed, exactness, simplicity, and cheapness. The thimble can be used a number of times.

Kalusky (Ananyst, 1913, 38, 48) also uses a thimble, but it cannot be raised or lowered. 